\title{
Adaptação e Validação Brasileira da Escala Older Workers' Intentions to Continue Working
}

\author{
Luciani Soares S. Macêdo ${ }^{1}$ \\ Pedro F. Bendassolli ${ }^{1}$ \\ Tatiana de Lucena Torres ${ }^{2}$ \\ Lucia Helena de Freitas Pinho França ${ }^{3}$ \\ ${ }^{1}$ Universidade Federal do Rio Grande do Norte \\ ${ }^{2}$ Universidade Federal da Paraíba \\ ${ }^{3}$ Universidade Salgado de Oliveira
}

\begin{abstract}
Resumo
Este artigo apresenta a adaptação e validação da escala Older Workers' Intentions to Continue Working (OWICW) em uma amostra de trabalhadores mais velhos de uma instituição de ensino superior do nordeste brasileiro. Participaram 283 servidores com idades entre 48 e 69 anos $(M=56,8 ; D P=5,1)$ com média de tempo de trabalho de 34 anos $(D P=4,4)$. Os dados foram submetidos à análise fatorial exploratória resultando em seis fatores com estrutura similar a cinco fatores do estudo original. As propriedades psicométricas sugerem a adequação da escala, que pode ser um instrumento útil à gestão de trabalhadores com mais idade que desejam continuar trabalhando. Sugere-se a continuidade dos estudos com outras populações.

Palavras-chave: trabalho, significado do trabalho, trabalhadores mais velhos, servidores públicos
\end{abstract}

\section{Brazilian Adaptation and Validation of the Older Workers' Intentions to Continue Working Scale}

\begin{abstract}
This article presents the adaptation and validation of the scale Older Workers' Intentions to Continue Working (OWICW) in a sample of older workers from a northeastern Brazilian higher education institution. The study was conducted with 283 federal employees with ages between 48 and 69 years $(M=56.8 ; S D=5.1)$ with an average working time of 34 years $(S D=4.4)$. The data were submitted to exploratory factor analysis resulting in six factors with a structure similar to five factors of the original study. The psychometrics properties suggest the adequacy of the scale, which can be a useful instrument to the management of older workers who wish to continue working. We suggest further studies with other populations.

Keywords: work; meaning of work; older workers; public service.
\end{abstract}

Adaptación y Validación Brasileña de la Escala Older Workers’ Intentions to Continue Working

\begin{abstract}
Resumen
Este artículo presenta la adaptación y validación de la escala Older Worker's Intentions to Continue, Working)OWICW) en una muestra de trabajadores mayores, de una institución de Enseñanza Superior, del nordeste brasileño. Participaron 283 funcionarios públicos con edades entre 48 y 69 años $(\mathrm{M}=56,8 ; \mathrm{DP}=5,1)$ con promedio de trabajo de 34 años $(\mathrm{DP}=4,4)$. Los datos fueron sometidos a análisis factorial exploratorio, resultando seis factores con estructura similar a cinco factores del estudio original. Las propiedades psicométricas indicaron la adecuación de la escala, que puede ser un instrumento útil para la gestión de trabajadores con más edad, que desean seguir trabajando. Se sugiere la continuidad de los estudios con otras poblaciones. Palabras clave: trabajo; significado de trabajo; trabajadores mayores; funcionarios públicos
\end{abstract}

\section{Introdução}

Nas últimas décadas, o fenômeno do envelhecimento e o declínio da proporção de trabalhadores ativos pela redução da natalidade se tornou uma das preocupações mundiais, sobretudo pelas possíveis consequências que anunciam, tais como a escassez da mão de obra, a lentificação do crescimento econômico e a insustentabilidade do sistema previdenciário. Governos e organizações dos países membros da Organização de Cooperação para Desenvolvimento Econômico (OECD) têm acionado políticas de geração de emprego para favorecer a retenção ou reinserção de trabalhadores mais velhos no mercado de trabalho, visando conter os efeitos negativos dessa realidade. Dentre essas políticas, cita-se o aumento da idade para elegibilidade de pensões públicas; as políticas ativas de reciclagem, treinamento e realocação; e o combate ao preconceito contra a idade (idadismo ou ageísmo) (e.g., Cooke, 2006; França, 2012; Gomes \& Pamplona, 2014),

Lidando com a transição demográfica há mais tempo, os países da OECD implementaram políticas consideradas referências no mundo. Para o Brasil, que recentemente formalizou um pedido de adesão à OECD (Chagas, 2017), a adoção de novas políticas e práticas laborais e de emprego é um desafio premente, 
pois o crescimento da população idosa está ocorrendo de forma mais acelerada do que em sociedades mais desenvolvidas (Camarano, Kanso, \& Fernandes, 2014; Zanelli, Silva, \& Tordera, 2013). O financiamento internacional de análises do panorama brasileiro, com vista a influenciar a formulação e reformulação de políticas públicas, ratifica que esse é um assunto vital e de interesse global (e.g., Gragnolati, Jorgensen, Rocha, \& Fruttero, 2011).

Paralelamente ao envelhecimento populacional, destaca-se o envelhecimento dos trabalhadores do serviço público que requer medidas urgentes de gestão de pessoas. Devido à rápida expansão dos serviços públicos em décadas anteriores, estima-se que, em treze países membros da OECD, mais de 30\% dessa força de trabalho deva se aposentar nos próximos 15 anos. No Brasil, foi observado que metade dos servidores já se encontra acima dos 50 anos e próximos da aposentadoria (OECD, 2009, 2010; Salomão, 2017).

Esse cenário atual aponta para uma participação crescente de trabalhadores mais velhos em um mercado de trabalho que, pelas mudanças sociais e avanços tecnológicos, está em constante mutação. Apesar disso, as iniciativas organizacionais brasileiras para se ajustar a essa realidade ainda são tímidas (Amorim, Fevorini, Melo, \& Tavares, 2016; Zanelli et al., 2013). Estudos apontam que a dificuldade de se dedicar a outros espaços de vida pessoal interfere na continuidade laboral, enquanto que um maior nível educacional ou o papel de provedor da família se relaciona com essa permanência (e.g., Camarano et al., 2014; França \& Carneiro, 2009; Macêdo, Bendassolli, \& Torres, 2017). Políticas que favoreçam a atualização e educação permanente do trabalhador, o equilíbrio trabalho/vida fora do trabalho, ou mesmo o combate ao idadismo nos ambientes organizacionais ainda são incipientes (França et al., 2014; França, 2012; Siqueira-Brito, França, \& Valentini, 2016; Stepansky \& França, 2008). Como observado por França et al., (2014), as medidas destinadas aos trabalhadores mais velhos concentram-se na progressão continuada na carreira e em medidas ergonômicas.

No Brasil, nota-se o início de uma discussão multidisciplinar sobre a relação entre o envelhecimento e o mercado de trabalho, ou sobre a atividade e capacidade laboral de trabalhadores mais velhos (e.g., Amorim et al., 2016; Amorim, Salla, \& Trelha, 2014; Gomes \& Pamplona, 2014; Siqueira-Brito et al., 2016). Mas, o interesse central no âmbito científico e do mercado de trabalho ainda circula na compreensão do que os jovens pensam e desejam em relação ao trabalho, pouco se conhecendo sobre as necessidades dos trabalhadores com mais idade (França, Menezes, Bendassolli, \& Macêdo, 2013; Zanelli et al., 2013). Os estudos para esta população estão voltados para o planejamento da aposentadoria (e.g., França \& Carneiro, 2009), a exceção de Menezes e França (2012) e Macêdo, Bendassolli e Torres (2019) que identificaram preditores da decisão de continuar trabalhando.

Diante da escassez de ferramentas que contribuam para entender o que os trabalhadores mais velhos esperam do trabalho, o objetivo do estudo aqui apresentado foi realizar uma adaptação transcultural da escala Older Workers' Intentions to Continue Working (OWICW), de Shacklock e Brunetto (2011). A escala OWICW, descrita na próxima seção deste artigo, foi originalmente construída visando obter informações que auxiliassem estratégias organizacionais de retenção de trabalhadores mais velhos em países da OCDE. Testando uma extensão do modelo de significado do trabalho (MOW, 1987; Westwood \& Lok, 2003), a escala se propõe a esclarecer uma de suas variáveis consequentes - as expectativas subjetivas sobre situações futuras de trabalho, que abrangem a importância de trabalhar e características intrínsecas do trabalho atrativas para esse grupo de trabalhadores. Como resultado na amostra australiana, os achados indicaram que a intenção de continuar trabalhando está significativamente relacionada às seguintes variáveis: percepção de autonomia no trabalho, a importância do trabalho para o indivíduo, a flexibilidade no trabalho, fatores administrativos e organizacionais, e interesses fora do trabalho. Entretanto, somente as últimas quatro variáveis se mostraram preditoras dessa intenção em uma análise de regressão múltipla (Shacklock \& Brunetto, 2011).

A intenção de manter-se trabalhando nessa fase de vida mais madura pressupõe a apreciação de valores que tornam o trabalho atrativo, interessante e gratificante, que se relaciona ao significado do trabalho para o indivíduo (Macêdo et al., 2019; MOW, 1987; Shacklock \& Brunetto, 2011). O modelo Meaning of Work (MOW) é reconhecidamente um dos mais influentes nesse campo de investigação. Mas, ainda que a produção científica brasileira sobre os sentidos e significados do trabalho tenha crescido nas últimas décadas, adaptando o MOW ou outros modelos teóricos, os estudos realizados não se voltam à especificidade dos trabalhadores mais velhos (e.g., Bendassolli, Alves, \& Torres, 2014; Bendassolli, Coelho-Lima, Pinheiro, \& Gê, 2015).

Estudos com jovens evidenciam que a concepção deles com o trabalho está associada principalmente

Psico-USF, Bragança Paulista, v. 25, n. 1, p. 127-138, jan./mar. 2020 
à dimensão econômica e a realização pessoal (e.g., Dutra-Thomé \& Koller, 2014; Fernandes, Gonçalves, \& Oliveira, 2012). Considerando que, com o passar do tempo o significado do trabalho passa por mudanças (Fraccaroli, 1994), e que fatores diversos podem explicar a intenção de permanecer trabalhando em diferentes estágios da carreira (Hellemans \& Closon, 2013), qual a função da atividade laboral para trabalhadores já próximos à aposentadoria? O propósito deste artigo é, por meio da adaptação transcultural e validação brasileira da escala OWICW, contribuir para a diversificação dos contextos de investigação e discutir sobre o que os trabalhadores com mais idade esperam do trabalho, a partir de uma amostra de servidores públicos de uma universidade federal.

\section{Método}

\section{Instrumento da Pesquisa: A Escala OWICW}

A versão original da OWICW tem 41 afirmativas, tipo Likert, com opções de respostas quanto ao grau de concordância de 1 (discordo totalmente) para 7 (concordo totalmente). A maior parte das afirmativas foi elaborada a partir do MOW (1987) e se refere a um conjunto de sete variáveis relativas ao trabalho: vínculo, importância do trabalho para o indivíduo, relacionamentos interpessoais, percepção de autonomia, flexibilidade, gestão e fatores organizacionais (aspectos do ambiente e relação supervisor-subordinado) e interesses fora do trabalho. Além dessas dimensões, a escala também inclui afirmações sobre saúde, finanças e dados sociodemográficos.

A validação original ocorreu com uma amostra de 379 trabalhadores acima dos 50 anos, pertencentes a uma grande organização do setor público situada em Queensland, Austrália. Os resultados do $\operatorname{KMO}(0,84)$ e o teste de Bartllet significativo $(\phi<0,001)$ indicaram a adequação da amostra. A escala apresentou propriedades psicométricas confiáveis (alfa de Cronbach $\geq$ $0,70)$ tendo os índices de confiabilidade confirmados os itens e a estrutura fatorial proposta de sete dimensões (Shacklock \& Brunetto, 2011). Não foram evidenciadas informações psicométricas de outras aplicações, nem de seu uso em contexto brasileiro. A escala foi disponibilizada pelas autoras e submetida primeiramente a uma adaptação transcultural.

\section{Procedimentos de Adaptação Transcultural}

Os procedimentos de adaptação envolveram inicialmente duas traduções realizadas separadamente por dois profissionais bilíngues. A qualidade das versões foi comparada com a versão original por um comitê proficiente em inglês, construindo uma versão síntese. Não se percebeu discrepância entre as duas traduções. Como grande parte das afirmativas do instrumento original estavam construídas gramaticalmente na voz passiva, houve a precaução de se preservar esse formato aproximando as afirmativas à forma brasileira de se expressar.

$\mathrm{Na}$ sequência, a versão síntese foi submetida à back translation por um terceiro tradutor bilíngue. $\mathrm{O}$ resultado foi utilizado como ferramenta de comparação ao instrumento original, por dois juízes experts independentes, bilíngues e especialistas. Os juízes revisaram todo o processo de adaptação, avaliando a equivalência semântica, idiomática, experiencial e conceitual de cada item, concordando com a maioria e sugerindo a revisão de alguns, enriquecendo o processo.

O instrumento foi submetido a um pré-teste com 15 sujeitos, com graus diversos de escolaridade e características similares ao público-alvo, buscando avaliar a compreensão das instruções, dos itens ou possíveis dificuldades. O tempo médio de preenchimento foi de 18 minutos. O fato da maioria dos itens da escala utilizar a mesma frase inicial "Minha intenção de continuar no trabalho remunerado seria influenciada por/ pelo(a)..." foi apontado como monótono. Como resultado do pré-teste, houve modificações ou acréscimos de alguns termos evitando entendimento diverso. Por exemplo, o termo "rendimento reduzido" foi substituído por "rendimento financeiro reduzido" evitando associação com a redução do desempenho. Por fim, após as modificações sugeridas, a escala foi considerada adequada à aplicação.

\section{Participantes}

\section{Critérios de Inclusão}

A terminologia "older workers" não é clara na definição da idade que demarca um trabalhador mais velho. $\mathrm{Na}$ Austrália, a idade mínima de aposentadoria é de 65 anos e o estudo de Shacklock e Brunetto (2011) utilizou uma amostra de trabalhadores acima de 50 anos. Em países nórdicos, critérios estatísticos apresentam a idade a partir de 45 anos (Tikkanen \& Nyhan, 2006). Neste estudo, considerando o regime especial de previdência dos servidores estatutários em vigor (ano de 2013), foram incluídos servidores ativos que já tivessem adquirido direito à aposentadoria ou se encontrassem até cinco anos antes de se aposentar. 


\section{Amostra}

A amostra, intencional e por conveniência, foi formada por 283 trabalhadores de uma instituição federal de ensino superior da região Nordeste do Brasil, sendo 36,4\% docentes e 63,6\% técnico-administrativos (TAs) de cargos diversos. A média de idade da amostra foi de 56,8 anos $(D P=5,1)$ e a média do tempo de contribuição era de 34 anos $(D P=4,4)$. Dentre os 283 respondentes, $61,5 \%$ eram mulheres e 38,5\% eram homens e $62,5 \%$ já faziam jus à aposentadoria, recebendo abono de permanência. O nível de escolaridade era elevado com $71 \%$ tendo cursado alguma pós-graduação e $65 \%$ declararam ter um parceiro ou cônjuge.

\section{Procedimentos}

\section{Coleta de Dados}

Os participantes foram convidados individualmente, via e-mail pessoal fornecido pela instituição. O instrumento foi disponibilizado por meio de um suporte on-line após declaração de consentimento aos objetivos e termos da pesquisa. O estudo foi aprovado pelo Comitê de Ética, CAAE 08938412.6.0000.5537, seguindo a todos os aspectos éticos e diretrizes dispostas na Resolução CNS 466/12. Ao término, uma devolutiva da pesquisa foi encaminhada aos participantes.

\section{Análise de Dados}

Os questionários sem missing $(n=283)$ foram considerados válidos e inseridos em uma planilha do software SPSS 20. Os dados foram submetidos a análises descritivas para avaliação das características dos itens da escala, detecção de erros e possíveis outliers univariados. A forma da distribuição de frequência se mostrou negativamente assimétrica. Nenhum dos outliers em potencial encontrados excedeu o critério referente ao escore-z (> 3,29), sugerido por Field (2009), não sendo deletado nenhum caso.

Por se tratar da primeira aplicação da escala OWICW em contexto brasileiro, a análise das propriedades psicométricas foi realizada por meio de uma análise fatorial exploratória (AFE) com dupla finalidade: verificar se os itens se agrupariam conforme previsto teoricamente e quais os índices de confiabilidade das dimensões (alfa de Cronbach). O método de extração escolhido foi a Fatoração dos Eixos Principais, recomendada para distribuições não normais. Assumindo a priori que as dimensões eram correlacionadas, foi utilizada a rotação Oblíqua (direct Oblimin), optando pela supressão dos itens com carga fatorial inferior a 0,4 (Laros, 2005).
Por fim, análises descritivas apontam as tendências da amostra nos fatores extraídos e estima-se o relacionamento destes com a intenção de continuar trabalhando usando o coeficiente de correlação de Pearson. Os escores de cada sujeito por fator foram estimados pela média dos pontos atribuídos aos itens. Variáveis demográficas (sexo, cargo e escolaridade) foram utilizadas para caracterizar a amostra e segmentá-la, explorando diferenças entre grupos (teste U de Mann-Whitney). Para isso, os TAs participantes foram categorizados em duas classes pela concentração do grau de escolaridade apresentado: TA de nível A, B e C $(n=52)$, com escolaridade de nível fundamental a médio, e TA de nível D e $\mathrm{E}(n=128)$, de superior incompleto a especialização. Para todas as análises realizadas, considerou-se como nível de significância $p<0,05$.

\section{Resultados}

Nesta seção, em primeiro lugar, apresentam-se os resultados referentes às propriedades psicométricas da versão brasileira da escala OWICW, descrevendo, em seguida, os fatores encontrados e seu relacionamento com a intenção de seguir trabalhando. Posteriormente, caracteriza-se a amostra apresentando as diferenças encontradas entre grupos.

\section{Propriedades Psicométricas da Escala OWICW - Versão Brasileira}

Como ponto de partida, foram considerados apenas os 31 itens do estudo original de validação de Shacklock e Brunetto (2011). Essas autoras excluíram os itens referentes à saúde e a finanças, justificando o objetivo de priorizar fatores menos investigados no contexto de trabalhadores mais velhos. Neste estudo, tendo observado que os valores da correlação item-total corrigida da subescala saúde apresentavam falta de consistência interna $(<0,3)$ e que os alfas de Cronbach das subescalas saúde $(\alpha=0,42)$ e finanças $(\alpha=0,46)$ indicavam baixa confiabilidade, optou-se também por excluí-los da AFE.

Os testes de fatorabilidade confirmaram a execução da análise fatorial pelo indicador Kaiser-Meyer-Olkin $(\mathrm{KMO}=0,83)$ e o teste de Bartlett significativo $\left(\chi^{2}=\right.$ $3882,83, p<0,001)$. O valor do determinante da matriz de correlação foi menor que 0,00001 levantando a hipótese da existência de multicolinearidade nos dados. No entanto, a inspeção da matriz não revelou variáveis com coeficientes altos $(>0,9)$ o que indicaria maior gravidade (Field, 2009) e a análise fatorial foi realizada. 
A primeira análise sugeriu a presença de nove fatores primários com eigenvalues superiores a 1 , pesos fatoriais iguais ou superiores a 0,40 , explicando $68,5 \%$ da variância. O screeplot mostrava dois pontos de inflexão (Figura 1): um mais acentuado no fator quatro e outro mais discreto no fator nove. Observou-se que os dois últimos fatores apresentavam baixa consistência e os cinco últimos foram definidos por um ou dois itens, não atendendo os critérios estabelecidos. A utilização de análise paralela de Horn, como uma estratégia adicional nessa decisão, fez observar que a sugestão parcimoniosa de quatro fatores efetuava a agregação inconsistente de alguns itens com sobrepujamento e perda de informações.

Considerando essas divergências, decidiu-se por realizar novas rotações, solicitando menos fatores em busca de uma solução que mantivesse a coerência teórica e não sacrificasse informações importantes. O critério de escolha da solução final dependia de cada fator conter um número mínimo de três itens contribuindo unicamente naquele fator, apresentando ainda boa consistência interna (Tabachnick \& Fidell, 2006).

A estrutura fatorial que se mostrou mais adequada neste estudo, segundo os critérios já explicitados, foi agrupada em seis fatores constituído por 24 itens dos 31 originais, explicando 58,55\% da variância total. Essa escala final de seis fatores correspondeu a cinco fatores da escala original, fez sentido intuitivo e apresentou alfa geral de 0,87. O Fator 6 considerado aceitável em cenários de investigação das ciências sociais ou em subescalas constituída por poucos itens. Todos os outros fatores apresentaram alfa igual ou

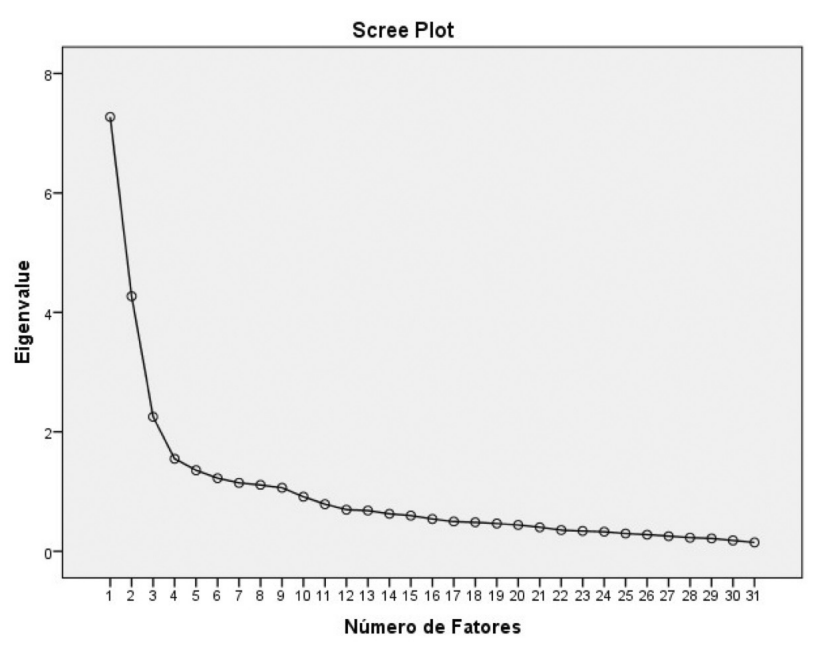

Figura 1. Diagrama de declividade. superior a 0,80 sendo um bom indicador de consistência interna e da propriedade psicométrica satisfatória da escala, legitimando o seu uso (Field, 2009). A maioria dos itens apresentou cargas fatoriais altas indicando seu grau de correlação com o fator. Os fatores encontrados serão descritos a seguir conforme a ordem que aparecem na Tabela 1.

Do ponto de vista descritivo, a Percepção de autonomia pessoal no trabalho foi o fator que sobressaiu com maior média, explicando $24 \%$ da variância. Esse fator foi composto por três itens de cinco do fator original. Para a maioria dos respondentes $(63,6 \%)$, a liberdade de colocar em prática as próprias ideias e decidir como fazer o seu trabalho influencia na intenção de continuar trabalhando.

Em seguida, o fator Relacionamento interpessoal no trabalho obteve a concordância de pouco mais da metade dos participantes (53,4\%), sendo o segundo com maior média. O fator foi formado pelos mesmos itens da subescala original. Esses itens avaliam a influência de benefícios alcançados por meio do contato social (relaxamento, descontração, diversão, distração dos problemas, valorização e convívio) sobre a intenção da continuidade do trabalho.

O terceiro fator, Interesses fora do trabalho, ficou composto por cinco dos sete itens da subescala original. Os itens investigam se outras áreas da vida (desenvolvimento espiritual, interesses comunitários, lazer, hobbies, amigos) influenciam sobre a continuidade do trabalho. A maioria dos respondentes $(63,6 \%)$ discorda que os interesses fora do trabalho afetam as intenções deles continuarem trabalhando.

O quarto fator, Flexibilidade no trabalho, foi formado por quatro itens. Apenas 29\% dos respondentes concordaram que a flexibilidade seria uma influência para a continuidade do trabalho. Um item do fator autonomia (A4) obteve carga significativa e ficou agregado nesse fator. O padrão de covariação desse item "caso eu pudesse ser meu próprio chefe" o associa mais à flexibilidade, o relacionando a ideia de fazer os próprios horários sem precisar dar satisfação aos outros. Observando os itens individualmente, 60\%declararam que poder controlar as horas e o modo de organização do trabalho e $51 \%$ que oportunidade de trabalhar meio período teria uma influência considerável. Mas, a oportunidade de trabalhar ocasionalmente não foi desejada pela maioria.

O quinto fator, Influência familiar, surgiu inesperadamente. Dois itens remanescentes da subescala original "interesses fora do trabalho" referentes ao 
Tabela 1.

Estrutura Fatorial da Versão Brasileira da Escala OWICW

\begin{tabular}{|c|c|c|c|c|c|c|}
\hline \multirow{2}{*}{ Itens sumarizados } & \multicolumn{6}{|c|}{ Fatores } \\
\hline & F1 & $\mathrm{F} 2$ & F3 & F4 & F5 & F6 \\
\hline (A1) Decidir como fazer meu trabalho & 0,87 & & & & & \\
\hline (A2) Colocar em prática minhas ideias & 0,75 & & & & & \\
\hline (A5) A liberdade para determinar como & 0,57 & & & & & \\
\hline (R4) Relaxamento e descontração que eu sinto & & 0,81 & & & & \\
\hline (R3) Não penso em outros problemas & & 0,78 & & & & \\
\hline (R1) Prazer e diversão que eu sinto & & 0,76 & & & & \\
\hline (R6) Oportunidade de lidar com pessoas & & 0,70 & & & & \\
\hline (R2) Sentimento de valorização & & 0,69 & & & & \\
\hline (R5) Não sinto solidão & & 0,61 & & & & \\
\hline (I5) Desenvolvimento espiritual & & & 0,76 & & & \\
\hline (I7) Interesses comunitários & & & 0,73 & & & \\
\hline (I6)Lazer & & & 0,70 & & & \\
\hline (I3) Hobbies & & & 0,62 & & & \\
\hline (I4) Amigos & & & 0,57 & & & \\
\hline (F2) Trabalhar meio-período & & & & 0,79 & & \\
\hline (F3) Controlar e escolher minhas horas & & & & 0,73 & & \\
\hline (F1) Trabalhar ocasionalmente & & & & 0,62 & & \\
\hline (A4) Ser meu próprio chefe & & & & 0,50 & & \\
\hline (I1) Parceiro ou cônjuge & & & & & 0,77 & \\
\hline (I2) Interesses familiares & & & & & 0,77 & \\
\hline (V1) Verdadeira vocação para o trabalho & & & & & & 0,71 \\
\hline (V3) Dedicação das pessoas na profissão & & & & & & 0,64 \\
\hline (V2) Rendimentos financeiros reduzidos & & & & & & 0,44 \\
\hline (IT1) Trabalhar é mais importante & & & & & & 0,43 \\
\hline Eigenvalues & 7,2 & 4,2 & 2,2 & 1,5 & 1,3 & 1,2 \\
\hline Variância Explicada\% & 24 & 13,9 & 7,3 & 5 & 4,3 & 4 \\
\hline$\alpha$ & 0,83 & 0,89 & 0,83 & 0,80 & 0,84 & 0,69 \\
\hline$M$ & 4,95 & 4,83 & 3,2 & 4 & 3,2 & 4,7 \\
\hline$D P$ & 1,55 & 1,35 & 1,45 & 1,65 & 1,9 & 1,2 \\
\hline$M d$ & 5,33 & 5 & 5 & 4 & 3 & 4,75 \\
\hline
\end{tabular}

Nota. Casos válidos: 283. Método de Extração: Fatoração de Eixos Principais. Método de rotação: Oblimin com normalização Kaiser. $\alpha=$ alfa de Cronbach 
tema família formaram um fator separado. De início, um item que originalmente pertencia ao fator administrativo e organizacional também se agregou. Essa combinação parecia ter o sentido de investigar o papel dos relacionamentos familiares e de relacionamentos negativos no ambiente de trabalho sobre a intenção da continuidade. No entanto, examinando a consistência interna desse novo fator, verificou-se que a remoção do item sobre o ambiente de trabalho foi indicada aumentando a confiabilidade de 0,67 para 0,84.

Embora esse fator tenha sido definido somente por duas variáveis, conforme Tabachnick e Fidell (2006), o fato de apresentarem alta correlação entre si $(r>0,70)$ e alta consistência interna $(\alpha=0,84)$ credita a ele confiabilidade. A maioria dos respondentes $(57,6 \%)$ discorda sobre a existência de uma influência familiar sobre a intenção de continuar trabalhando.

O fator Vínculo com o trabalho sofreu uma alteração da escala original recebendo um item que anteriormente pertencia à Subescala importância do trabalho: "Trabalhar é a coisa mais importante da minha vida". Os itens que o compõem procuram investigar sobre a importância e a identificação com o trabalho. Esse fator é o terceiro com média mais elevada. Apenas $48 \%$ dos respondentes opinaram sobre sua influência positiva. Os achados ainda indicam que a intenção de continuar no trabalho remunerado está significativamente relacionada a nível $p<0,01$ com os fatores Autonomia, Relacionamento interpessoal, Interesses fora do trabalho e Vínculo com o trabalho; e a nível $p<$ 0,05 com o fator Influência familiar.

\section{Variáveis Sociodemográficas e Fatores Relacionados ao Trabalho}

Análises bivariadas com o teste $U$ de Mann-Whitney revelaram diferenças significativas entre os respondentes. Os docentes consideram a percepção da autonomia pessoal no trabalho um fator mais atrativo para a continuidade laboral do que os técnico-administrativos $A-B-C$ que possuem grau de escolaridade mais baixa $(U=2249,50 ; r=-0,13)$. Esse efeito foi relatado $\operatorname{com} p=0,049$ (unicaudal), significância testada com o método de Monte Carlo, apresentando IC 99\% [0,44; $0,55]$. Por outro lado, os técnico-administrativos de nível $A-B-C$ consideram o relacionamento interpessoal no trabalho um fator mais influente do que os docentes $(U=1836,00 ; r=-0,25)$. Esse efeito foi relatado ao nível $p<0,01$.

Também foi observado que a percepção de influência dos interesses fora do trabalho sobre a intenção de continuar trabalhando difere entre cargos e entre os sexos. Os técnico-administrativos D-E discordam mais do que os técnico-administrativos $A-B-C$ quanto à influência desse fator $(U=2598,50 ; r=-0,17)$, sendo essa diferença estatisticamente significativa a $p=0,018$ (bicaudal). Semelhantemente, as mulheres discordam mais do que os homens quanto à percepção de influência desse fator $(U=7563,50 ; r=-0,17)$. Esse efeito foi relatado $p<0,01$ (bicaudal).

Por sua vez, os servidores homens concordam mais do que as mulheres quanto à percepção de influência do vínculo com o trabalho sobre a intenção de continuar trabalhando $(U=8383,50 ; p<0,049$ (uni caudal) significância testada com o método de Monte Carlo, apresentando IC 99\% [0,43; 0,54]. Quanto à percepção de influência familiar, as mulheres discordam mais do que os homens $(U=6957,50 ; r=-0,27)$ a respeito da influência desse fator sobre a continuidade laboral. Semelhantemente, os participantes que não têm parceiros discordam mais do que os casados $(U=$ 5321,50; $r=-0,35)$. Esses últimos efeitos foram relatados como significativos a $p<0,001$ (bicaudal).

\section{Discussão}

O presente estudo teve por objetivo realizar uma adaptação transcultural da escala Older Workers' Intentions to Continue Working (OWICW), como uma ferramenta para identificar características do trabalho que são atrativas para trabalhadores mais velhos. Os resultados corroboraram o modelo de Shacklock e Brunetto (2011) em cinco, dos sete fatores originais (Vínculo com o trabalho, Interesses fora do trabalho, Percepção de autonomia pessoal, Flexibilidade e Interesses fora do trabalho), apresentando propriedades psicométricas satisfatórias para o uso em contexto brasileiro. No entanto, semelhantemente a Shacklock e Brunetto, as correlações entre os fatores e a intenção de continuar trabalhando variaram de intensidade fraca a moderada. Mesmo assim, não seria de se esperar correlações elevadas para uma variável que tem se mostrado multideterminada, como apontado por França, Menezes, Bendassolli e Macêdo (2013). Não obstante, algumas diferenças e considerações merecem ser destacadas, ainda que em uma perspectiva qualitativa e exploratória.

$\mathrm{Na}$ amostra australiana, os resultados da análise fatorial da escala mostram que a maior parte da variância explicada foi respondida pelo fator Interesses fora do trabalho $(24,2 \%)$, Flexibilidade $(11,5 \%)$ e Fatores administrativos e organizacionais $(9,2 \%)$. Porém, na brasileira, são os fatores Autonomia (24\%), seguido do fator Relacionamentos interpessoais (14\%) e Interesses fora do trabalho $(7 \%)$ que concentram maior 
porcentagem. Sendo ambas as amostras provenientes de organizações públicas, essa diferença entre os países pode se atribuir ao fato de que, na cultura brasileira, a percepção de autonomia e a interação no ambiente de trabalho são aspectos mais valorizados nessa fase de vida, considerando-se trabalhadores de uma instituição de educação superior.

A percepção pessoal de autonomia no trabalho foi mais valorizada por cargos com maior grau de escolaridade e maior complexidade de tarefas. Esse resultado corrobora com Menezes e França (2012) que, em um estudo com servidores da área de ciência e tecnologia, identificaram que os dois maiores preditores para a permanência dos trabalhadores mais velhos na instituição foram a autonomia (nomeada também de controle no trabalho) associada à flexibilidade. Amorim et al., (2016) também observou uma relação entre o nível de escolaridade alta e a permanência no mercado de trabalho. Vazquez, Magnan, Pacico, Hutz e Schaufeli (2015) encontraram relação significativa entre o engajamento no trabalho e certos grupos ocupacionais com maior autonomia na condução de suas atividades e observaram que esse engajamento aumenta de forma ascendente e significativa com a idade. Esses dados mostram a relevância do estímulo à educação permanente e a atualização dos trabalhadores mais velhos, proporcionando oportunidades para o desenvolvimento pessoal e da carreira, como também apontado por França et al., (2014).

Quanto ao fator Relacionamentos interpessoais, que não sofreu alteração da escala original, destaca-se para esses trabalhadores a função atrativa do trabalho de oportunizar experiências interpessoais satisfatórias, que contribui para o significado dado ao trabalho. $\mathrm{O}$ combate ao ageísmo e a adoção de estratégias facilitadoras de integração dos grupos intergeracionais ajudaria nesse aspecto, a exemplo de Siqueira-Brito, França e Valentini (2016).

Todos os itens do fator interesses fora do trabalho permaneceram na validação brasileira, mas esse fator se desmembrou em dois, gerando outro que foi denominado de "influência familiar". O posicionamento da família como um fator separado sugere que, a respeito do espaço de não trabalho, a esfera familiar se distingue do nível dos outros interesses e espaços de vida pública e pessoal. Esse pode ser um aspecto cultural importante já que, em outros estudos transculturais, a influência dos relacionamentos familiares tem surgido como um diferencial brasileiro, como apontado por França (2012). Esse achado também pode ter relação com características da amostra utilizada, já que a maioria declarou ter um parceiro ou cônjuge. Dentre os homens casados, $21 \%$ eram os provedores únicos do lar, o que possivelmente esclareça o fato da percepção dessa influência ser maior para eles.

Vale destacar ainda que os relacionamentos familiares e os outros interesses (amigos, lazer, hobbies etc.) comumente são citados como tornando mais provável a decisão de aposentadoria (França et al., 2013; Macêdo et al., 2019; Macêdo et al., 2017). Todavia, a relevância dos interesses fora do trabalho surge em ambas as populações como um aspecto importante a se considerar quanto à intenção de continuar trabalhando nessa fase.

Dois dos itens originais do fator Importância do trabalho e os quatro itens relativos ao fator Administrativo e organizacional não demonstraram um bom desempenho nessa adaptação. Esses itens foram suprimidos na AFE pelo critério de valor da carga fatorial, o que pode revelar algum problema dessa medida. Para os australianos, esses dois fatores se mostraram preditores da intenção de continuidade laboral, embora o fator Importância do trabalho tenha obtido a menor média dentre os outros $(M=2,7 ; D P=1,35)$ (Shacklock \& Brunetto, 2011).

Observando-se o posicionamento dos brasileiros quanto ao fator importância do trabalho, embora a maioria $(80 \%)$ tenha declarado que "trabalhar é a coisa mais importante da minha vida" (IT1) e que continuaria trabalhando mesmo que ganhasse uma grande quantia na loteria ou de uma herança (63\%), a percepção de importância do trabalho decresce bastante (33\%) ao ser comparada com outras áreas de vida (família, amigos, comunidade, lazer etc.). O lugar dado ao trabalho, em comparação a outras áreas, e o surgimento do fator relativo à família sugerem que há uma delimitação marcante das principais esferas de vida. Investir em medidas educativas que orientem ao equilíbrio entre essas esferas poderia ajudar na retenção dos trabalhadores dessa faixa etária (Stepansky \& França, 2008).

O primeiro desses itens (IT1) terminou por se agregar no fator vínculo com o trabalho, unindo um aspecto da centralidade ao envolvimento afetivo. Além de esse fator estar significativamente relacionado com a intenção de continuar trabalhando, é mais valorizado pelo sexo masculino, correspondendo ainda com o papel tradicional de gênero. Por outro lado, Amorim et al., (2016) observou um maior vínculo empregatício dos trabalhadores homens acima dos 50 anos associado com a estabilidade oferecida pelo serviço público. 
Já com respeito ao fator Administrativo, questões relacionadas à burocracia organizacional, à chefia, ao ambiente de trabalho e à capacidade física foram vistas pelos australianos como um fator que afetaria a intenção de continuidade laboral. $\mathrm{Na}$ amostra brasileira, elas não são percebidas como relevantes, talvez devido ao contexto institucional observado. Mas, a maioria reconhece que um ambiente de trabalho desagradável $(60 \%)$ ou diminuição da capacidade física para desenvolver a atividade $(76 \%)$ afetaria a intenção de continuar trabalhando. Esses últimos itens parecem contrastar com a valorização dada aos relacionamentos positivos no trabalho e à autonomia pessoal, comentados anteriormente.

Quanto à flexibilidade, embora tenha sido apontada como um aspecto desejado do trabalho por Shacklock e Brunetto (2011), Menezes e França (2012) e Zanelli, Silva e Tordera, (2013), neste estudo, não surgiu como uma condição significativa. Uma possível explicação para isso é que a maioria dos participantes já se encontrava em situação de adiamento da aposentadoria, podendo ter se reportado à experiência atual de trabalho em que a flexibilidade de horário não se configurava uma possibilidade.

O modelo australiano aponta que arranjos de trabalho flexíveis que possibilitem atender aos interesses fora do trabalho, oportunidades de desenvolvimento pessoal e a gestão e supervisão dos ambientes de trabalho seriam medidas de retenção dos trabalhadores acima de 50 anos. Já o modelo brasileiro sugere que o estímulo à educação permanente oportunizando o desenvolvimento pessoal e da carreira, a promoção de boas relações no ambiente de trabalho e a orientação para o equilíbrio entre as esferas de vida seriam as medidas mais adequadas. Para essa faixa etária, os dados sugerem que os objetivos e resultados valorizados do trabalho surgem como aspectos mais relevantes no significado do trabalho do que a centralidade.

\section{Considerações Finais}

Em síntese, este estudo indica que a versão brasileira da escala OWICW apresenta propriedades psicométricas satisfatórias. Nesse sentido, contribui com uma ferramenta que pode ser útil à gestão de pessoas para a compreensão das necessidades dos trabalhadores com mais idade. No entanto, algumas limitações precisam ser mencionadas, o que pode motivar o interesse de outros pesquisadores em dar continuidade ao tema.
Em primeiro lugar, há possibilidade de condicionamento dos resultados em função do tipo de público investigado: majoritariamente profissionais com nível de escolaridade elevado, incluindo professores, pertencentes a uma instituição pública de ensino superior. Não por acaso, uma das dimensões mais importantes apontadas pelo instrumento é a autonomia, compreensível pelas características do trabalho nesse contexto. Embora se possa assumir a hipótese que a estrutura fatorial do construto permaneceria consistente, independentemente de uma mudança nesse cenário, outros fatores poderiam se destacar ao se tentar explicar a intenção de continuar trabalhando com trabalhadores de instituições/organizações privadas.

Novos estudos poderiam explorar, não só o contexto, como também a influência do tipo de atividade sobre as variáveis preditoras da continuidade do trabalho. Especialmente debruçando-se sobre variáveis relativas ao sentido do trabalho. Por exemplo, o trabalho de um professor poderia ser internamente mais recompensador, pelo menos em termos simbólicos, que muitas outras atividades, no amplo espectro de ocupações, que incluam tarefas extremamente repetitivas ou que exijam intenso desgaste físico. Estas são questões que podem enriquecer as dimensões que constituem o significado do trabalho e suas relações com a intenção de trabalhar ou aposentar-se.

Em segundo lugar, com relação ao plano metodológico, têm-se as limitações de alcance impostas por um estudo inicial, utilizando uma estratégia exploratória de análise. Uma sugestão seria ampliar a aplicação do instrumento para outros públicos, inclusive comparando-os em termos de momentos na trajetória da carreira, de modo a se ter condições de realizar uma análise confirmatória. Embora, ao longo deste texto, tenha-se explorado as similaridades e distanciamentos desta validação com o estudo australiano original, um desenho confirmatório, calibrando as diferenças ao nível dos itens, aprofundaria a compreensão das convergências e divergências existentes. Além disso, outros aspectos culturais, além da cultura organizacional em questão (setor de instituição de ensino superior pública) poderiam ser igualmente verificados em um desenho com essa orientação.

Ainda que se considerem tais limitações, o estudo vem preencher lacunas do processo de avaliação com trabalhadores mais velhos, no contexto de investigação sobre a relação trabalho-aposentadoria, como também destaca a importância de características do trabalho que exercem influência para a retenção de trabalhadores 
no mercado de trabalho. É claro que a intenção é um terreno multifatorial, e as influências são múltiplas. Contudo, no caso da intenção de continuidade do trabalho ou decisão pela aposentadoria, não se pode minimizar, como parece ser o caso em diversos estudos ainda hoje, o sentido intrínseco do trabalho realizado, bem como suas conexões com outros domínios de vida. O trabalho ocupa um papel indiscutível na estruturação das experiências de tempo, carreira e futuro da maior parte das pessoas. Por isso, fica o convite para que os pesquisadores de ambos os "campos", significados do trabalho, de um lado, e aposentadoria, de outro, passem a conceber esse fenômeno a partir de suas inevitáveis conexões.

\section{Referências}

Amorim, W. A. C., Fevorini, F. B., Melo, A. F. S., \& Tavares, A. S. (2016). O mercado de trabalho para trabalhadores com 50 anos ou mais no Brasil. Boletim de Informações Fipe, No. 435. Recuperado de http://downloads.fipe.org.br/content/downloads/publicacoes/bif/bif435-9-24.pdf

Amorim, J. S. C., Salla, S., \& Trelha, C. S. (2014). Fatores associados à capacidade para $\mathrm{O}$ trabalho em idosos: Revisão sistemática. Revista Brasileira de Epidemiologia, 17(4), 830-841. doi:10.1590/1809-4503201400040003

Bendassolli, P. F., Alves, J. S. C., \& Torres, C. C. (2014). Inventário sobre significado do trabalho de profissionais de indústrias criativas. Avaliação Psicológica, 13(2), 177-186. Recuperado de http://www.redalyc.org/html/3350/335031819005/

Bendassolli, P. F., Coelho-Lima, F., Pinheiro, R. A., \& Gê, P. C. S. (2015). The Brazilian scientific production on sense and meaning of work: Review of use of terminology and current thematic classifications. Avances em Psicología Latino americana, 33(2), 203-221. doi:10.12804/ap133.02.2015.03

Camarano, A. A., Kanso, S., \& Fernandes, D. (2014). Menos jovens e mais idosos no mercado de trabalho. Em A. A. Camarano (Org.), Novo Regime Demográfico: Uma nova relação entre população e desenvolvimento? (pp. 377-406). Rio de Janeiro: IPEA.

Chagas, P. V. (2017). Brasil formaliza pedido de adesão à OCDE. Agência Brasil. Recuperado de http:/ /agenciabrasil.ebc.com.br/economia/noticia/2017-05/ brasil-formaliza-pedido-de-adesao-ocde
Cooke, M. (2006). Policy changes and the labour force participation of older workers: Evidence from six countries. Canadian Journal on Aging/La revue canadienne du vieillissement, 25(4), 387-400. doi: 10.1353/ cja.2007.0015

Dutra-Thomé, L., \& Koller, S. H. (2014). O significado do trabalho na visão de jovens brasileiros: Uma análise de palavras análogas e opostas ao termo "trabalho". Revista Psicologia Organizações e Trabalho, 14(4), 367-380. Recuperado de http://pepsic.bvsalud.org/pdf/rpot/v14n4/v14n4a04.pdf

Fernandes, F. S., Gonçalves, C. M., \& Oliveira, P. J.(2012). Adaptação e validação da Escala de Significados Atribuídos ao Trabalho - ESAT. Revista Brasileira de Orientação Profissional,13(2), 183-195. Recuperado de http://pepsic.bvsalud.org/pdf/ rbop/v13n2/06.pdf

Field, A. (2009). Descobrindo a estatística usando o SPSS. Porto Alegre: Artmed.

Fraccaroli, F. (1994). Stabilité et changement des significations du travail. Une recherche longitudinale sur la transition école-emploi. Le Travail Humain, 57(2), 145-162. Recuperado de http://www.jstor. org/stable/40659846

França, L. (2012). Envelhecimento dos trabalhadores nas organizações: Estamos preparados? Em L. França e D. Stepansky (Orgs.), Propostas multidisciplinares para o bem-estar na aposentadoria (pp. 25-52). Rio de Janeiro: Quartet/FAPERJ.

França, L. H. F. P., \& Carneiro, V. L. (2009). Programas de preparação para a aposentadoria: Um estudo com trabalhadores mais velhos em Resende (RJ). Revista Brasileira de Geriatria e Gerontologia, 12(3), 429-447. Recuperado de http://www.redalyc.org/ pdf/4038/403838782010.pdf

França, L. H. F. P., Menezes, G. S., Bendassolli, P. F., \& Macêdo, L. S. S. (2013). Aposentar-se ou continuar trabalhando? O que influencia essa decisão. Psicologia Ciência e Profissão, 33(3), 548-563. Recuperado de http://submission.scielo.br/index.php/pcp/ article/view/77508

França, L. H. F. P., Nalin, C. P., Siqueira-Brito, A. R., Amorim, S. M., Rangel. T. \& Eckman, N. C. (2014). A percepção dos gestores brasileiros sobre os programas de preparação para aposentadoria. Estudos Interdisciplinares sobre o Envelhecimento, 19(3), 
879-898. Recuperado de http://www.seer.ufrgs. br/RevEnvelhecer/article/view/50434

Gomes, P. S., \& Pamplona, J. B. (2014). Envelhecimento populacional, mercado de trabalho e políticas públicas de emprego nos países da OCDE. Pesquisa \& Debate, 25(2), 127-151. Recuperado de https://revistas.pucsp.br/index.php/rpe/article/ view/21495/15763

Gragnolati, M., Jorgensen. O. H., Rocha, R., \& Fruttero, A. (2011). Envelhecendo em um Brasil mais velho - Sumário Executivo. Washington, D.C: Banco Mundial. Recuperado de http://jornalggn.com.br/sites/ default/files/documentos/envelhecendo_brasil_ sumario_executivo.pdf

Hellemans, C., \& Closon, C. (2013). Intention to remain at work until legal retirement age: A comparative analysis among different age subgroups of employees. Europe's Journal of Psychology, 9(3), 623-639. doi:10.5964/ejop.v9i3.614

Laros, J. A. (2005). O uso da análise fatorial: Algumas diretrizes para pesquisadores. Em L. Pasquali, Análise fatorial para pesquisadores (pp. 163-184). Brasília, DF: Laboratório de Pesquisa em Avaliação e Medida.

Macêdo, L. S. S., Bendassolli, P. F., \& Torres, T. L. (2017). Representações sociais da aposentadoria e intenção de continuar trabalhando. Psicologia \& Sociedade, 29, 1-11. doi: 10.1590/1807-0310/2017v29145010

Macêdo, L. S. S., Bendassolli, P. F., \& Torres, T.L. (2019). Preditores do adiamento da aposentadoria por servidores públicos federais. Avances em Psicología Latinoamericana, 37(1), 153-167. doi: 10.12804/ revistas.urosario.edu.co/apl/a.4556

Menezes, G. S., \& França, L. H. (2012). Preditores da decisão da aposentadoria por servidores públicos federais. Revista Psicologia Organizações e Trabalbo, 12(3), 315-328. Recuperado de http://pepsic.bvsalud.org/pdf/rpot/v12n3/v12n3a06.pdf

MOW International Research Team. (1987). The meaning of working. London: Academic Press.

Organização de Cooperação e Desenvolvimento Econômico - OECD. (2009). Government at a Glance 2009. Paris: OECD Publishing. doi: 10.1787/9789264075061-en

Organização de Cooperação e Desenvolvimento Econômico - OECD. (2010). Avaliação da Gestão de Recursos Humanos no Governo - Relatório da OCDE: Brasil,
Governo Federal (Portuguese version). Paris: OECD Publishing. doi:10.1787/9789264086098-pt

Salomão, A. (2017). Quase 2 milhões de servidores podem se aposentar nos próximos dez anos. Estadão [Jornal digital], Recuperado em 09 de março, 2018, de http://economia.estadao.com.br/noticias/ geral,quase-2-milhoes-de-servidores-podem-se-aposentar-nos-proximos-dez-anos, 70001923600

Shacklock, K., \& Brunetto, Y. (2011). A model of older workers' intentions to continue working. Personnel Review, 40(2), 252-274. doi:10.1108/00483481111106110

Siqueira-Brito, A. D. R., França, L. H. F. P., \& Valentini, F. (2016). Análise fatorial confirmatória da Escala de Ageísmo no Contexto Organizacional. Avaliação Psicológica, 15(3), 337-345. doi:10.15689/ ap.2016.1503.06

Stepansky, D. V., \& França, L. (2008). Trabalho e vida pessoal: $\mathrm{O}$ equilíbrio necessário. Boletim Técnico do Senac, 34(1), 64-71. Recuperado de http://www. bts.senac.br/index.php/bts/article/view/282

Tabachnick, B. G., \& Fidell, L. S. (2006). Using multivariate statistics (5th ed.). Boston, MA: Allyn \& Bacon/ Pearson Education.

Tikkanen, T., \& Nyhan, B. (Eds.). (2006). Promoting lifelong learning for older workers: an international overview. Recuperado de www.cedefop.europa.eu/ files/3045_en.pdf

Westwood, R., \& Lok, P. (2003). The meaning of work in chinese contexts: A comparative study. International Journal of Cross Cultural Management, 3(2), 139-165. doi:10.1177/14705958030032001

Vazquez, A. C. S., Magnan, E. D. S., Pacico, J. C., Hutz, C. S., \& Schaufeli, W. B. (2015). Adaptation and validation of the Brazilian version of the Utrecht work engagement scale. Psico-USF, 20(2), 207-217. doi:10.1590/1413-82712015200202

Zanelli, J. C., Silva, N., \&Tordera, N. (2013). Orientação para aposentadoria e gestão de pessoas nas organizações. Em L. O. Borges \& L. Mourão (Orgs.), O trabalho e as organizações: Atuacões a partir da psicologia (pp. 644-668). Porto Alegre: Artmed.

Recebido em: 21/03/2018 Reformulado em: 18/09/2018 e 30/11/2018

Aceito: 24/03/2019 
Nota dos autores:

Manuscrito “Adaptação e Validação Brasileira da Escala Older Workers’ Intentions to Continue Working”.

Sobre os autores:

Luciani Soares S. Macêdo é Psicóloga, Doutora em Psicologia pela Universidade Federal do Rio Grande do Norte UFRN. Servidora da Pró-Reitoria de Gestão de Pessoas/UFRN. Membro da equipe de coordenação do "Projeto Vida com Maturidade" de orientação à aposentadoria e ao envelhecimento ativo desde 2003.

ORCID: https://orcid.org/0000-0003-0281-7703

E-mail:lucianissm@gmail.com

Pedro F. Bendassolli é Psicólogo, Doutor em Psicologia Social pela USP (2006). Estágios pós-doutorais na Université Paris IX (Centre de Recherche Management \& Organisation, 2008-2009), na UNB (2009-2012) e no Centre for Cultural Psychology - Aalborg University, Dinamarca, (2016-2017). Professor Adjunto do Departamento de Psicologia, Universidade Federal do Rio Grande do Norte. Atua na área da Psicologia do Trabalho e das Organizações.

ORCID: https://orcid.org/0000-0002-7761-0857

E-mail:pbendassolli@gmail.com

Tatiana de Lucena Torres é Professora do Departamento de Psicologia, Universidade Federal da Paraíba. Membro do Grupo Subjetividade e Trabalho. Membro da Rede Internacional de Pesquisa sobre Representação Social, Envelhecimento e Saúde (RIPRES).

ORCID: https://orcid.org/0000-0001-6274-1929

E-mail: tltorres2@gmail.com

Lucia Helena de Freitas Pinho França é Psicóloga, Doutora (PhD) em Psicologia pela Universidade de Auckland - NZ (2004), Mestre em Psicologia Social pela UFRJ (1989) e Gerontóloga pelo Instituto Sedes Sapientiae/SBGG-SP (1989). Professor titular do Programa de Pós-Graduação em Psicologia da UNIVERSO - Universidade Salgado de Oliveira (Niterói). Coordenou o Trabalho Social com Idosos no SESC - DN (1979-1995). É consultora em Programas de Preparação para a Aposentadoria (PPA).

ORCID: https://orcid.org/0000-0003-0676-3757

E-mail: luciafranca@luciafranca.com

Contato com os autores:

Luciani Soares S. Macêdo

(DAS/PROGESP/UFRN)

Av. Senador Salgado Filho, s/n, Campus Universitário

Caixa postal 1524,

Natal-RN, Brasil

CEP: 59078-970 